\title{
Observer-Based Vaccination Strategy for a True Mass Action SEIR Epidemic Model with Potential Estimation of All the Populations
}

\author{
M. De la Sen, ${ }^{1}$ A. Ibeas, ${ }^{2}$ and S. Alonso-Quesada ${ }^{1}$ \\ ${ }^{1}$ Institute of Research and Development of Processes, Faculty of Science and Technology, \\ University of the Basque Country, P. O. Box 644, 48940 Bilbao, Spain \\ ${ }^{2}$ Department of Telecommunications and Systems Engineering, Autonomous University of Barcelona, \\ 08193 Bellaterra, Barcelona, Spain
}

Correspondence should be addressed to M. De la Sen, manuel.delasen@ehu.es

Received 14 June 2011; Accepted 8 August 2011

Academic Editor: Vimal Singh

Copyright (C) 2011 M. De la Sen et al. This is an open access article distributed under the Creative Commons Attribution License, which permits unrestricted use, distribution, and reproduction in any medium, provided the original work is properly cited.

This paper presents a simple continuous-time linear vaccination-based control strategy for a SEIR (susceptible plus infected plus infectious plus removed populations) propagation disease model. The model takes into account the total population amounts as a refrain for the illness transmission since its increase makes more difficult contacts among the susceptible and infected. The control objective is the asymptotically tracking of the removed-by-immunity population to the total population while achieving simultaneously the remaining population (i.e., susceptible plus infected plus infectious) to asymptotically converge to zero. A state observer is used to estimate the true various partial populations of the susceptible, infected, infectious, and immune which are assumed to be unknown. The model parameters are also assumed to be, in general, unknown. In this case, the parameters are replaced by available estimates to implement the vaccination action.

\section{Introduction}

Important control problems nowadays related to life sciences are the control of ecological models like, for instance, those of population evolution (Beverton-Holt model, Hassell model, Ricker model, etc.) via the online adjustment of the species environment carrying capacity, that of the population growth, or that of the regulated harvesting quota as well as the disease propagation via vaccination control. In a set of papers, several variants and generalizations of the Beverton-Holt model (standard time-invariant, time-varying parameterized, generalized model or modified generalized model) have been investigated at the levels of stability, cycleoscillatory behavior, permanence, and control through the manipulation of the carrying 
capacity (see, e.g., [1-5]). The design of related control actions has been proved to be important in those papers at the levels, for instance, of aquaculture exploitation or plague fighting. On the other hand, the literature about epidemic mathematical models is exhaustive in many books and papers. A nonexhaustive list of references is given in this paper; compare [6-14] (see also the references listed therein). The sets of models include the most basic ones, [6, 7].

(i) SI-models where not removed-by-immunity population is assumed. In other words, only susceptible and infected populations are assumed.

(ii) SIR models, which include susceptible plus infected plus removed-by—immunity populations.

(iii) SEIR models where the infected populations is split into two ones (namely, the "infected" which incubate the disease but do not still have any disease symptoms and the "infectious" or "infective" which do have the external disease symptoms).

Those models have also two major variants, namely, the so-called "pseudo-mass action models," where the total population is not taken into account as a relevant disease contagious factor and the so-called "true-mass action models," where the total population is more realistically considered as an inverse factor of the disease transmission rates. There are many variants of the above models, for instance, including vaccination of different kinds as follows: constant $[8,9]$, impulsive $[9,12]$, discrete-time, and so forth, incorporating point or distributed delays $[12,13]$, oscillatory behaviours [14], and so forth. On the other hand, variants of such models become considerably simpler for the illness transmission among plants [6, 7]. Recent work on the influence of latent and infective periods and studiers of persistence of infection has been performed in $[15,16]$. Other mathematical models involving coupled partial populations of the whole ones require very close analysis of equilibrium points, stability, and positivity to that required in epidemic models (see, e.g., $[17,18]$ ).

In this paper, a continuous-time vaccination observer-based control strategy is given for a SEIR epidemic model which takes directly the estimated susceptible, infected, infectious, and immune populations to design the vaccination strategy. It is not required either the knowledge through time of the true partial populations of the susceptible, infected, infectious, and immune, since this task is performed by the observer, nor the knowledge of the true parameters. The incorporation of the observer to the vaccination control rule is the main contribution of this paper. On the other hand, it is assumed that the total population is known and equal to the total estimated population and also that it remains constant through time, so that the illness transmission is not critical, and the SEIR model is of the above-mentioned truemass action type. Some important issues of positivity, stability, and model reference tracking of a suitable population evolution of the combined SEIR model and its observer are discussed.

\subsection{Notation}

$\mathbf{R}_{+}^{n}$ is the first open $n$-real orthant, and $\mathbf{R}_{0+}^{n}$ is the first closed $n$-real orthant.

$x \in \mathbf{R}_{0+}^{n}$ is a positive real $n$-vector in the usual sense that all its components are nonnegative. This can be also denoted by $x>0$ if $x \neq 0$. If all the components are strictly positive, this is denoted by $x \gg 0$ or $x \in \mathbf{R}_{+}^{n}$. In the same way, $A \in \mathbf{R}_{0+}^{n \times n}$ is a positive real $n$-matrix in the usual sense that all its entries are nonnegative and we can use the notations $A>0$ and $A \gg 0$ (if $A \in \mathbf{R}_{+}^{n \times n}$ ). A square real matrix $A$ is a Metzler matrix if and only if all its off-diagonal entries are nonnegative and then its associate exponential matrix function is positive; that is, none of its entries takes a negative value at any time. 
$C^{(q)}(\mathrm{Do} ; \mathrm{Im})$ is the set of real functions of class $q$ of domain Do and image $\mathrm{Im}$. $P C^{(q)}(\mathrm{Do} ; \mathrm{Im})$ is the set of real functions of class $(q-1)$ of domain Do and image Im whose $q$ th derivative exits but it is not necessarily everywhere continuous on its definition domain.

\section{SEIR Epidemic Model}

Let $S(t)$ be the "susceptible" population of infection at time $t, E(t)$ the "infected" (i.e., those which incubate the illness but do not still have any symptoms) at time $t, I(t)$ is the "infectious" (or "infective") population at time $t$, and $R(t)$ is the "removed-by-immunity" (or "immune") population at time $t$. Consider the true-mass action SEIR-type epidemic model:

$$
\begin{gathered}
\dot{S}(t)=-\mu S(t)+\omega R(t)-\beta \frac{S(t) I(t)}{N}+\mu N(1-V(t)) \\
\dot{E}(t)=\beta \frac{S(t) I(t)}{N}-(\mu+\sigma) E(t) \\
\dot{I}(t)=-(\mu+\gamma) I(t)+\sigma E(t) \\
\dot{R}(t)=-(\mu+\omega) R(t)+\gamma I(t)+\mu N V(t)
\end{gathered}
$$

subject to initial conditions $S_{0}=S(0) \geq 0, E_{0}=E(0) \geq 0, I_{0}=I(0) \geq 0$, and $R_{0}=R(0) \geq 0$ under the constraint $N=N(0)=S(t)+E(t)+I(t)+R(t)=S(0)+E(0)+I(0)+R(0)$; for all $t \in \mathbf{R}_{0+}$ and the vaccination function $V: \mathbf{R}_{0+} \rightarrow \mathbf{R}_{0+}$. In the above SEIR model, $N$ is the total population, $\mu$ is the rate of deaths from causes unrelated to the infection, $\omega$ is the rate of losing immunity, $\beta$ is the transmission constant (with the total number of infections per unity of time at time $t$ being $\beta(S(t) I(t) / N)$ ), and $\sigma^{-1}$ and $\gamma^{-1}$ are finite and, respectively, the average durations of the latent and infective periods. All the above parameters are assumed to be nonnegative. Note that $S, E, I, R \in C^{(1)}\left(\mathbf{R}_{0+} ; \mathbf{R}\right)$ if $V \in C^{(0)}\left(\mathbf{R}_{0+} ; \mathbf{R}\right)$. However, if $V \in P C^{(0)}\left(\mathbf{R}_{0+} ; \mathbf{R}\right)$ then $S, E, I, R \in C^{(0)}\left(\mathbf{R}_{0+} ; \mathbf{R}\right)$, their time-derivatives exist but they are not everywhere continuous on $\mathbf{R}_{0+}$, in general. This model has been studied in [19] from the point of view of equilibrium points in the free-vaccination case and control. Two vaccination auxiliary controls (grouping the last three terms of the right-hand side of (2.1)) being, respectively, proportional to the susceptible or to the whole population so that the whole population is asymptotically immune have been proposed. Note that controllability is a very suitable property of practical control problems since each state variable is allowed to track a prescribed value, in a finite time (see, e.g., [20]). However, we have to point out that epidemic models are not, in general, controllable since all the partial populations cannot be separately controlled through vaccination since there is always an inherent interchange among the various populations [19]. The SEIR model (2.1)-(2.4) is uncontrollable according to the above principle so that there are potential final values of some of the partial populations which are not within the set of reachable states [21]. The epidemic models proposed in previous works assume that the parameters of the SEIR model are known. However, the research in this paper does not require such a perfect parametrical knowledge since an observer with parametrical estimation is incorporated. It has to be pointed out that the model following and tracking of desired references as well as the optimization of certain well-posed criteria are very important objectives in control theory problems including robotics and adaptive control [22-24]. They are also relevant in predator-prey processes or in epidemics as, for instance, the removal 
or asymptotic removal of diseases through vaccination strategies [25-28]. In this paper, the vaccination rules are based on the estimation of the partial populations provided by an "ad hoc" synthesized observer which is potentially useful in the common real case that not all partial populations which are integrated in the whole dynamic epidemic model are precisely known or measurable for all time.

\section{Observer-Based Vaccination Control Strategy for the SEIR Model}

It turns out that, while the assumption of the knowledge of the total population $N$ is not quite restrictive in practice, the knowledge of the individual various partial populations of the susceptible, infected, infectious, and immune may be considered severely restrictive. It is seen that if the partial initial populations are unknown then their evolution through time cannot be computed in a closed form from the differential system (2.1)-(2.4). A practical solution to circumvent the problem might be to estimate them based on percentages of the total population through time from experimental knowledge of the disease propagation. Another solution may be to estimate them online by using an online observer. The current paper focuses on this solution by using a SEIR-estimation algorithm (observer) of the SEIR model (2.1)-(2.4) which estimates through time the individual populations being involved. The vaccination strategy is obtained as a control strategy from the data supplied by the observer through time. Such a strategy does not require the knowledge of the partial populations to organize and perform the vaccination strategy. The estimates of the various individual populations are denoted by the same notations as the real populations with hat superscripts, namely, $\widehat{S}(t), \widehat{E}(t), \widehat{I}(t), \widehat{R}(t)$, Thus, consider the SEIR-type observer for the SEIR-epidemic model (2.1)-(2.4) as follows:

$$
\begin{gathered}
\dot{\hat{S}}(t)=-\widehat{\mu} \widehat{S}(t)+\widehat{\omega} \widehat{R}(t)-\widehat{\beta} \frac{\widehat{S}(t) \widehat{I}(t)}{N}+\widehat{\mu} N(1-V(t)) \\
\dot{\hat{E}}(t)=\widehat{\beta} \frac{\widehat{S}(t) \widehat{I}(t)}{N}-(\widehat{\mu}+\widehat{\sigma}) \widehat{E}(t) \\
\dot{\hat{I}}(t)=-(\widehat{\mu}+\widehat{\gamma}) \widehat{I}(t)+\widehat{\sigma} \widehat{E}(t) \\
\dot{\hat{R}}(t)=-(\widehat{\mu}+\widehat{\omega}) \widehat{R}(t)+\widehat{\gamma} \widehat{I}(t)+\widehat{\mu} N V(t)
\end{gathered}
$$

subject to initial conditions $\widehat{S}_{0}=\widehat{S}(0) \geq 0, \widehat{E}_{0}=\widehat{E}(0) \geq 0, \widehat{I}_{0}=\widehat{I}(0) \geq 0$, and $\widehat{R}_{0}=\widehat{R}(0) \geq 0$ under the constant population constraint

$$
N=N(0)=\widehat{S}(t)+\widehat{E}(t)+\widehat{I}(t)+\widehat{R}(t)=\widehat{S}(0)+\widehat{E}(0)+\widehat{I}(0)+\widehat{R}(0)
$$

equalizing its total estimated value for all time, for all $t \in \mathbf{R}_{0+}$ and the vaccination law $V: \mathbf{R}_{0+} \rightarrow \mathbf{R}_{0+}$ generated by

$$
V(t)=\frac{1}{\widehat{\mu} N}\left(k_{1} \widehat{S}(t)+k_{2} \widehat{E}(t)+k_{3} \widehat{I}(t)+k_{4} \widehat{R}(t)+k_{5} \widehat{S}(t) \widehat{I}(t)+g N\right)
$$

where $k_{i}, i=1,2, \ldots, 5$, are constant real gains to be determined in order to achieve the control objective. In the above estimated SEIR model, $\widehat{\mu}$ is the estimate of $\mu$, that is, the estimated rate 
of deaths from causes unrelated to the infection and also the estimated rate of births, $\widehat{\omega}$ is the estimate of $\omega$, that is, the estimated rate of losing immunity, $\widehat{\beta}$ is the estimate of $\beta$, that is, the estimated transmission constant (with the total number of infections per unity of time at time $t$ being $\widehat{\beta}(\widehat{S}(t) \widehat{I}(t) / N)$ ), and $\widehat{\sigma}^{-1}$ and $\widehat{\gamma}^{-1}$ are finite and, respectively, the estimates of $\sigma^{-1}$ and $\gamma^{-1}$, that is, the estimated average durations of the latent and infective periods, respectively. The estimations of the above parameters can be done, through the use of available "a priori" knowledge, to be identical to the true values if those ones are known or estimated online from data measurements. Through this paper, we assume that those estimated parameters are fixed but not necessarily identical to the true parameters and all of them are nonnegative. The substitution of (3.3) in (3.1) yields the following combined observer-controller for the SEIR model:

$$
\dot{\hat{x}}(t)=\widehat{A}(t) \widehat{x}(t)+\widehat{b},
$$

where

$$
\begin{gathered}
\widehat{x}(t):=(\widehat{S}(t), \widehat{E}(t), \widehat{I}(t), \widehat{R}(t))^{T}, \quad \widehat{b}:=((\widehat{\mu}-g) N, 0,0, g N)^{T} \\
\widehat{A}(t):=\left[\begin{array}{cccc}
-\left(\widehat{\mu}+k_{1}+\left(\widehat{\beta}_{1}+k_{5}\right) \widehat{I}(t)\right) & -k_{2} & -k_{3} & \widehat{\omega}-k_{4} \\
\widehat{\beta}_{1} \widehat{I}(t) & -(\widehat{\mu}+\widehat{\sigma}) & 0 & 0 \\
0 & \widehat{\sigma} & -(\widehat{\mu}+\widehat{\gamma}) & 0 \\
k_{1}+k_{5} \widehat{I}(t) & k_{2} & \widehat{\gamma}+k_{3} & -\left(\widehat{\mu}+\widehat{\omega}-k_{4}\right)
\end{array}\right], \widehat{\beta}_{1}:=\frac{\widehat{\beta}}{N} .
\end{gathered}
$$

The substitution of (3.3) into (2.1)-(2.4) yields the following SEIR observer-based vaccination controlled SEIR model:

$$
\dot{x}(t)=A(t) x(t)+B(t) \widehat{x}(t)+b,
$$

where

$$
\begin{gathered}
x(t):=(S(t), E(t), I(t), R(t))^{T} ; \quad b:=\left(\left(1-\frac{g}{\widehat{\mu}}\right) \mu N, 0,0, \frac{g \mu N}{\widehat{\mu}}\right)^{T} \\
A(t):=\left[\begin{array}{cccc}
-\left(\mu+\beta_{1} I(t)\right) & 0 & 0 & \omega \\
\beta_{1} I(t) & -(\mu+\sigma) & 0 & 0 \\
0 & \sigma & -(\mu+\gamma) & 0 \\
0 & 0 & \gamma & -(\mu+\omega)
\end{array}\right], \quad \beta_{1}:=\frac{\beta}{N}
\end{gathered}
$$




$$
B(t):=\left(\frac{\mu}{\widehat{\mu}}\right)\left[\begin{array}{cccc}
-\left(k_{1}+k_{5} \widehat{I}(t)\right) & -k_{2} & -k_{3} & -k_{4} \\
0 & 0 & 0 & 0 \\
0 & 0 & 0 & 0 \\
k_{1}+k_{5} \widehat{I}(t) & k_{2} & k_{3} & k_{4}
\end{array}\right] .
$$

The systems (3.4)-(3) and (3.7)-(3.10) may be compacted as an extended system as follows:

$$
\dot{\bar{x}}(t):=\bar{A}(t) \bar{x}(t)+\bar{b}
$$

with

$$
\bar{A}(t):=\left[\begin{array}{cc}
\widehat{A}(t) & 0 \\
A(t)-\widehat{A}(t)+B(t) & A(t)
\end{array}\right] ; \quad \bar{b}:=\left(\hat{b}^{T}, \tilde{b}^{T}\right)^{T},
$$

$\bar{x}(t):=\left(\widehat{x}^{T}(t), \tilde{x}^{T}(t)\right)^{T}$ with $\tilde{x}(t)=x(t)-\widehat{x}(t)$ being the observation error and $\tilde{b}$ is a parametrical error defined by

$$
\tilde{b}:=b-\widehat{b}=\left(\frac{\mu}{\widehat{\mu}}-1\right) N(\widehat{\mu}-g, 0,0, g)^{T}
$$

It is direct to see that $\|\tilde{b}\| \leq \varepsilon$ for any given real $\varepsilon>0$, with $\|\tilde{b}\|:=|(\mu / \widehat{\mu})-1| N \sqrt{(\widehat{\mu}-g)^{2}+g^{2}}$ being the Euclidean norm of $\tilde{b}$, if $|\mu-\widehat{\mu}| \leq \widehat{\mu} \varepsilon / N \sqrt{(\widehat{\mu}-g)^{2}+g^{2}}$. Decompose

$$
A(t):=A_{0}+\Delta A(t) ; \quad \widehat{A}(t):=\widehat{A}_{0}+\Delta \widehat{A}(t) ; \quad A(t)-\widehat{A}(t)+B(t)=B_{0}+\Delta B(t),
$$

where $A_{0}, \widehat{A}_{0}$, and $B_{0}$ are constant matrices being the nonunique decompositions (3.14) as follows:

$$
\begin{aligned}
A_{0} & :=\left[\begin{array}{cccc}
-\left(\mu+\beta_{1} I_{r}\right) & 0 & 0 & \omega \\
0 & -(\mu+\sigma) & 0 & 0 \\
0 & \sigma & -(\mu+\gamma) & 0 \\
0 & 0 & \gamma & -(\mu+\omega)
\end{array}\right] ; \\
\Delta A(t) & :=\left[\begin{array}{cccc}
\beta_{1}\left(I_{r}-I(t)\right) & 0 & 0 & 0 \\
\beta_{1} I(t) & 0 & 0 & 0 \\
0 & 0 & 0 & 0 \\
0 & 0 & 0 & 0
\end{array}\right]
\end{aligned}
$$




$$
\begin{aligned}
& \widehat{A}_{0}:=\left[\begin{array}{cccc}
-\left(\widehat{\mu}+k_{1}+\left(\widehat{\beta}_{1}+k_{5}\right) \widehat{I}_{r}\right) & -k_{2} & -k_{3} & \widehat{\omega}-k_{4} \\
0 & -(\widehat{\mu}+\widehat{\sigma}) & 0 & 0 \\
0 & \widehat{\sigma} & -(\widehat{\mu}+\widehat{\gamma}) & 0 \\
k_{1}+k_{5} \widehat{I}_{r} & k_{2} & \widehat{\gamma}+k_{3} & -\left(\widehat{\mu}+\widehat{\omega}-k_{4}\right)
\end{array}\right] ; \\
& \Delta \widehat{A}(t):=\left[\begin{array}{cccc}
\left(\widehat{\beta}_{1}+k_{5}\right)\left(\widehat{I}_{r}-\widehat{I}(t)\right) & 0 & 0 & 0 \\
\widehat{\beta}_{1} \widehat{I}(t) & 0 & 0 & 0 \\
0 & 0 & 0 & 0 \\
k_{5}\left(\widehat{I}(t)-\widehat{I}_{r}\right) & 0 & 0 & 0
\end{array}\right] \\
& \Delta B(t)=A(t)-\widehat{A}(t)+B(t)-B_{0} \\
& =\left[\begin{array}{cccc}
M & \left(1-\frac{\mu}{\widehat{\mu}}\right) k_{2} & \left(1-\frac{\mu}{\widehat{\mu}}\right) k_{3} & \omega-\widehat{\omega}+\left(1-\frac{\mu}{\widehat{\mu}}\right) k_{4} \\
\beta_{1} I(t)-\widehat{\beta}_{1} \widehat{I}(t)-b_{021} & \widehat{\mu}-\mu+\widehat{\sigma}-\sigma & 0 & 0 \\
0 & \sigma-\widehat{\sigma} & \widehat{\mu}+\widehat{\gamma}-\mu-\gamma & 0 \\
-\left(1-\frac{\mu}{\widehat{\mu}}\right)\left(k_{1}+k_{5} \widehat{I}(t)\right) & -\left(1-\frac{\mu}{\widehat{\mu}}\right) k_{2} & \gamma-\widehat{\gamma}-\left(1-\frac{\mu}{\widehat{\mu}}\right) k_{3} & \widehat{\mu}+\widehat{\omega}-\mu-\omega-\left(1-\frac{\mu}{\widehat{\mu}}\right) k_{4}
\end{array}\right],
\end{aligned}
$$

where $\mathcal{M}$ denotes the term " $\widehat{\mu}-\mu+\widehat{\beta}_{1} \widehat{I}(t)-\beta_{1} I(t)+(1-(\mu / \widehat{\mu}))\left(k_{1}+k_{5} \widehat{I}(t)\right)-b_{011}$ " for any given prefixed constant values $I_{r} \geq 0, \widehat{I}_{r} \geq 0$ and $B_{0}:=\left[\begin{array}{cccc}b_{01} & 0 & 0 & 0 \\ b_{221} & 0 & 0 \\ 0 & 0 & 0 & 0 \\ 0 & 0 & 0 & 0\end{array}\right]$ so that $\bar{A}(t)$ is decomposed as follows:

$$
\bar{A}(t)=\bar{A}_{0}+\tilde{\bar{A}}_{0}(t) ; \quad \bar{A}_{0}:=\left[\begin{array}{cc}
\widehat{A}_{0} & 0 \\
B_{0} & A_{0}
\end{array}\right] ; \quad \widetilde{\bar{A}}_{0}(t):=\left[\begin{array}{cc}
\Delta \widehat{A}(t) & 0 \\
\Delta B(t) & \Delta A(t)
\end{array}\right] .
$$

If $A_{0}$ and $\widehat{A}_{0}$ are stability (or Hurwitz) matrices then the block triangular matrix $\bar{A}_{0}$ is a stability matrix with stability abscissa $(-\rho)$ which is subject to $\max \left(\operatorname{Re} \lambda_{i}\left(A_{0}\right), \operatorname{Re} \lambda_{i}\left(\widehat{A}_{0}\right)\right) \leq-\rho<0$, where the first inequality is nonstrict if there is some multiple eigenvalue of $\bar{A}_{0}$.

\section{About the Stability of the SEIR Extended Epidemic Model (3.11)-(3.16)}

The SEIR epidemic model (2.1)-(2.4) and its observer (3.4)-(3) are both always globally stable if $N<\infty$ if the initial real and observed populations are bounded and equalize $N$ provided that both systems are positive as, on the other hand, the real problem at hand requires. This would imply that all the particular populations (susceptible, infected, infectious, and immune) are nonnegative and less than $N$ so that they are uniformly bounded for all time. Note that if any of the populations of the SEIR model is negative then boundedness of all 
the components of the associated system is not guaranteed. The boundedness of all the partial populations upperbounded by a bounded total population $N$ guarantees that the extended SEIR system (3.11)-(3.16), constructed with the estimated and observer error states, is stable with $\sum_{i=1}^{4} \widehat{x}_{i}(t)=N$ and Euclidean norm $\|\widetilde{x}(t)\| \leq\|x(t)\|+\|\widehat{x}(t)\| \leq 2 \sqrt{2} N$. This fact does not guarantee by itself that $\bar{A}(t)$ is a stability matrix function, that the observation error converges asymptotically to zero, or that, at least, it converges to a small residual set around zero. This feature merits further discussion. Note that simple inspection shows that $A_{0}$ is a stability matrix if $d(s)=\left(s+\mu+\beta_{1} I_{r}\right)(s+\mu+\sigma)(s+\mu+\gamma)(s+\mu+\omega)$ is Hurwitz. Also, $\widehat{A}_{0}$ is a stability matrix if $\operatorname{det}\left(s I-\widehat{A}_{0}\right)=\widehat{d}(s)+\left(k_{1}+k_{5} \widehat{I}_{r}\right) \widehat{n}(s)$ has all its zeros in $\operatorname{Re} s<0$, where

$$
\begin{gathered}
\widehat{n}(s)=\left(k_{4}-\widehat{\omega}\right)(s+\widehat{\mu}+\widehat{\sigma})(s+\widehat{\mu}+\widehat{\gamma}) \\
\widehat{d}(s)=\left(s+\widehat{\mu}+k_{1}+\left(\widehat{\beta}_{1}+k_{5}\right) \widehat{I}_{r}\right)(s+\widehat{\mu}+\widehat{\sigma})(s+\widehat{\mu}+\widehat{\gamma})\left(s+\widehat{\mu}+\widehat{\omega}-k_{4}\right) .
\end{gathered}
$$

Assume that $\widehat{d}(s)$ is a Hurwitz polynomial, that is,

$$
k_{4}<\widehat{\mu}+\widehat{\omega}, \quad \widehat{\mu}+k_{1}+\left(\widehat{\beta}_{1}+k_{5}\right) \widehat{I}_{r}>0, \quad \widehat{\mu}+\widehat{\sigma}>0, \quad \widehat{\mu}+\widehat{\gamma}>0
$$

and define $\widehat{h}(s):=\left(k_{1}+k_{5} \widehat{I}_{r}\right) \widehat{n}(s) / \widehat{d}(s)$. Note that

$$
\operatorname{det}\left(s I-\widehat{A}_{0}\right)=\widehat{d}(s)+\left(k_{1}+k_{5} \widehat{I}_{r}\right) \widehat{n}(s)=\widehat{d}(s)(1+\widehat{h}(s))=0 \Longleftrightarrow 1+\widehat{h}(s)=0
$$

has all its solutions in $\operatorname{Re} s<0$ for all $\widehat{n}(s)$ of the form (4.1) from the small gain theorem if and only if $\|\widehat{h}\|_{\infty}:=\sup _{\omega \in \mathbf{R}_{0+}}|\widehat{n}(\mathbf{i} \omega) / \widehat{d}(\mathbf{i} \omega)|<1(\mathbf{i}=\sqrt{-1}$ being the imaginary complex unit) since $\widehat{d}(s)$ is a Hurwitz polynomial and where $\|\widehat{h}\|_{\infty}$ is the $H_{\infty}$-norm of the transfer function $\widehat{h}(s)$. Since $\bar{A}_{0}$ is blocktriangular and constant then the following result is direct.

Assertion 4.1. $\bar{A}_{0}$ is a stability matrix if and only if $\mu+\beta_{1} I_{r}>0, \mu+\sigma>0, \mu+\gamma>0, \mu+\omega>0$ and $\widehat{h} \in \mathbf{R H}_{\infty}$ (i.e., $\left.k_{4}<\widehat{\mu}+\widehat{\omega} ; \widehat{\mu}+k_{1}+\left(\widehat{\beta}_{1}+k_{5}\right) \widehat{I}_{r}>0 ; \widehat{\mu}+\widehat{\sigma}>0 ; \widehat{\mu}+\widehat{\gamma}>0\right)$ with $\|\widehat{h}\|_{\infty}<1$.

From Assertion 4.1 and Gronwall's Lemma [29] the following follows.

Assertion 4.2. The matrix function $\bar{A}(t)$ is stable if $\bar{A}_{0}$ is a stability matrix, that is, if (4.3) holds and, furthermore, $\rho>\sup _{t \in \mathbf{R}_{0+}}\left\|\tilde{\bar{A}}_{0}(t)\right\|$, where $(-\rho)<0$ is the stability abscissa of the (stability) matrix $\bar{A}_{0}$.

Another alternative sufficiency type stability condition of $\bar{A}(t)$, which replaces the constraint $\rho>\sup _{t \in \mathbf{R}_{0+}}\left\|\tilde{\bar{A}}_{0}(t)\right\|$ in Assertion 4.2 (see [30] for close related problems), is as follows.

Assertion 4.3. The matrix function $\bar{A}(t)$ is stable, so that the unforced extended system (3.11)(3.16) is then globally exponentially stable, if $\bar{A}_{0}$ is a stability matrix (see Assertion 4.1) and, furthermore, there exist norm-dependent real constants $\alpha_{0} \in \mathbf{R}_{0+}$ (being sufficiently small) and $\alpha_{1} \in \mathbf{R}_{0+}$ such that $\int_{t}^{t+T}\left\|\dot{\overline{\bar{A}}}_{0}(\tau)\right\| d \tau \leq \alpha_{0} T+\alpha_{1}$ for any given fixed $T \in \mathbf{R}_{+} ;$for all $t \in \mathbf{R}_{0+}$. 
Note that the Euclidean norm of $\bar{b}$ may be directly calculated from those of $\widehat{b}$ and $\tilde{b}$ using (3) and (3.13) leading to

$$
\|\bar{b}\| \leq \frac{(\widehat{\mu}+|\mu-\widehat{\mu}|) N}{\widehat{\mu}} \sqrt{(\widehat{\mu}-2 g) \widehat{\mu}+2 g^{2}} \leq \frac{(2 \widehat{\mu}+\mu) N}{\widehat{\mu}} \sqrt{(\widehat{\mu}-2 g) \widehat{\mu}+2 g^{2}}
$$

since $\|\widehat{b}\|=N \sqrt{(\widehat{\mu}-2 g) \widehat{\mu}+2 g^{2}}$ and $\|\tilde{b}\|=(|\mu-\widehat{\mu}| N / \widehat{\mu}) \sqrt{(\widehat{\mu}-2 g) \widehat{\mu}+2 g^{2}}$. Note that $\rho_{0}:=$ $\rho-\sup _{t \in \mathbf{R}_{0+}}\left\|\tilde{\bar{A}}_{0}(t)\right\|>0$ so that $\left(-\rho_{0}\right)<0$ is larger than the maximum of the stability abscissas of $\bar{A}(t)$ for $t \in \mathbf{R}_{0+}$ if Assertion 4.3 holds.

Assertion 4.4. If Assertion 4.3 holds then any solution of the forced system (3.11) to (3.16) satisfies the following inequality for some real constant $k_{0} \geq 1$ :

$$
\begin{aligned}
\|\bar{x}(t)\| & \leq M(t):=k_{0} e^{-\rho_{0} t}\left(\|\bar{x}(0)\|+\|\bar{b}\| \int_{0}^{t} e^{\rho_{0} \tau} d \tau\right) \\
& \longrightarrow \frac{k_{0}}{\rho_{0}} \frac{(\widehat{\mu}+|\mu-\widehat{\mu}|) N}{\widehat{\mu}} \sqrt{(\widehat{\mu}-2 g) \widehat{\mu}+2 g^{2}} \text { as } t \longrightarrow \infty,
\end{aligned}
$$

and the corresponding substates of $\bar{x}(t)$ satisfy

$$
\begin{aligned}
\|\widehat{x}(t)\| & \leq \widehat{M}(t):=k_{0} e^{-\rho_{0} t}\left(\|\widehat{x}(0)\|+\|\widehat{b}\| \int_{0}^{t} e^{\rho_{0} \tau} d \tau\right) \\
& \longrightarrow \frac{k_{0}}{\rho_{0}} N \sqrt{(\widehat{\mu}-2 g) \widehat{\mu}+2 g^{2}} \text { as } t \longrightarrow \infty \\
\|\widetilde{x}(t)\| & \leq \widetilde{M}(t):=k_{0} e^{-\rho_{0} t}\left(\|\tilde{x}(0)\|+\|\tilde{b}\| \int_{0}^{t} e^{\rho_{0} \tau} d \tau\right) \\
& \longrightarrow \frac{k_{0}}{\rho_{0}} \frac{|\mu-\widehat{\mu}| N}{\widehat{\mu}} \sqrt{(\widehat{\mu}-2 g) \hat{\mu}+2 g^{2}} \text { as } t \longrightarrow \infty .
\end{aligned}
$$

Note that $\|\widetilde{\mathbf{x}}(\mathbf{t})\| \rightarrow \mathbf{0}$ as $\mathbf{t} \rightarrow \infty$ if $\widehat{\boldsymbol{\mu}}=\boldsymbol{\mu}$ (and then $\|\widehat{\mathbf{x}}(\mathbf{t})\| \rightarrow\left(\mathbf{k}_{0} \mathbf{N} / \rho_{0}\right) \sqrt{(\boldsymbol{\mu}-\mathbf{2 g}) \boldsymbol{\mu}+2 \mathbf{g}^{2}}$ as $\mathbf{t} \rightarrow \infty)$ and, if in addition, $\mathbf{g}=\mathbf{0}$ then $\|\widehat{\mathbf{x}}(\mathbf{t})\| \rightarrow \mathbf{k}_{0} \mu \mathbf{N} / \rho_{0}$ as $\left.\mathbf{t} \rightarrow \infty\right)$ or if $\widehat{\boldsymbol{\mu}}=\mathbf{g}=\mathbf{0}$ (and then $\|\widehat{\mathbf{x}}(\mathbf{t})\| \rightarrow \mathbf{0}$ as $\mathbf{t} \rightarrow \infty)$. Since $\mathbf{b}:=((\mathbf{1}-\mathbf{g} / \widehat{\boldsymbol{\mu}}) \boldsymbol{\mu} \mathbf{N}, \mathbf{0}, \mathbf{0}, \mathbf{g} \boldsymbol{\mu} \mathbf{N} / \widehat{\boldsymbol{\mu}})^{\mathrm{T}}$ then $\|\mathbf{x}(\mathbf{t})\| \leq$ $\|\mathbf{M}(\mathbf{t})\| \rightarrow\left(\mathbf{k}_{0} / \rho_{0}\right)(\widehat{\mu}+|\boldsymbol{\mu}-\widehat{\boldsymbol{\mu}}| \mathbf{N} / \widehat{\boldsymbol{\mu}}) \sqrt{(\widehat{\mu}-2 \mathbf{g}) \widehat{\boldsymbol{\mu}}+\mathbf{2 g}^{2}}$ as $\mathbf{t} \rightarrow \infty$. However, this upperbound can be improved if a version of Assertion 4.3 applied to the matrix function $\mathbf{A}(\mathbf{t})$ leads to a smaller ratio of its corresponding constants than the ratio $k_{0} / \rho_{0}$ of the whole extended system. 


\section{About the Positivity of the SEIR Extended Epidemic Model (3.11)-(3.16)}

Positive systems are those having nonnegative solutions in the sense that all the state components are nonnegative for all time [31,32]. Because of the nature of the SEIR epidemic model (2.1)-(2.4), it is required that it be a positive system for the implemented vaccination law. The extended SEIR system has a unique solution for each initial state given by:

$$
\bar{x}(t)=e^{\bar{A}_{0} t}\left(\bar{x}(0)+\int_{0}^{t} e^{-\bar{A}_{0} \tau}\left(\widetilde{\bar{A}}_{0}(\tau) \bar{x}(\tau)+\bar{b}\right) d \tau\right) .
$$

From (3.4) and (3.7) the SEIR solution and its estimate through the observer are uniquely given by

$$
\begin{gathered}
\widehat{x}(t)=e^{\widehat{A}_{0} t}\left(\widehat{x}(0)+\int_{0}^{t} e^{-\widehat{A}_{0} \tau}(\Delta \widehat{A}(\tau) \widehat{x}(\tau)+\widehat{b}) d \tau\right) \\
x(t)=e^{A_{0} t}\left(x(0)+\int_{0}^{t} e^{-A_{0} \tau}[(\Delta A(\tau) x(\tau)+B(\tau) \widehat{x}(\tau)+b)] d \tau\right) .
\end{gathered}
$$

In principle, it is apparently nonnecessary to require in addition that the estimation algorithm or the extended system be positive. However, note the following features by direct inspection of (5.2)-(5.3).

(1) If $\widehat{A}_{0}$ is a Metzler matrix, $\Delta \widehat{A}(t)>0$, for all $t \in \mathbf{R}_{0+}$ and $\widehat{b}>0$, then $\widehat{x}(0)>0 \Leftrightarrow$ $\widehat{x}(t)>0$, for all $t \in \mathbf{R}_{0+}$.

(2) If $\widehat{x}(t)>0$, for all $t \in \mathbf{R}_{0+}$ (i.e., if $\widehat{A}_{0}$ is a Metzler matrix, $\Delta A(t)>0$; for all $t \in \mathbf{R}_{0+}$, $\widehat{b}>0$ and $\widehat{x}(0)>0), b>0, \Delta A(t)>0, B(t)>0$, for all $t \in \mathbf{R}_{0+}$ and $A_{0}$ is a Metzler matrix then $\left(x^{T}(0), \widehat{x}^{T}(0)\right)^{T}>0 \Rightarrow x(t)>0$, for all $t \in \mathbf{R}_{0+}$.

(3) $\widehat{A}_{0}$ depends on the vaccination gain $k_{1}, \Delta \widehat{A}(t)$ does not depend on $k_{1}$, but it depends on $k_{5}$ (see (3.15)), and $B(t)$ in (3.10) depends on $k_{i}(i=1, \ldots, 5)$. Thus, if $k_{1}$ is chosen so that $\widehat{A}_{0}$ is not a Metzler matrix (because its (4.1) entry is negative), $\widehat{x}(t)$ may be nonpositive at some time for some nonnegative initial condition $\widehat{x}(0)$. At the same time, the vector function $B(t) \widehat{x}(t)$ may have some sufficiently negative component at some time " $t$ " so that some corresponding component of $x(t)$ may be negative at that time. A close reasoning may be used for the case that $k_{5}$ is such that $B(t)$ is nonpositive (even if $\widehat{A}_{0}$ is a Metzler matrix).

The following result follows from the above observations.

Assertion 5.1. The following properties hold.

(i) Assume that $A_{0}$ and $\widehat{A}_{0}$ are Metzler matrices, $\Delta A(t)>0, b+B(t) \widehat{x}(t) \geq 0, \Delta \widehat{A}(t)>0$, for all $t \in \mathbf{R}_{0+}, b>0$ and $\widehat{b}>0$ then $\left(x^{T}(0), \widehat{x}^{T}(0)\right)^{T}>0 \Rightarrow x(t)>0, \widehat{x}(t)>0$, for all $t \in \mathbf{R}_{0+}$. In other words, the extended system of state $\left(x^{T}(t), \hat{x}^{T}(t)\right)^{T}$ is positive. 
(ii) Assume that in Property (i) $\hat{A}_{0}$ fails to be a Metzler matrix because of the value $k_{1}$ in its $(4,1)$ entry of $\Delta \widehat{A}(t)$ is not positive due to the parameter $k_{5}$. Then, for initial conditions $\widehat{x}(0)>0$ which make $\widehat{x}(t)$ be nonpositive (i.e., with some negative component on a time interval), $x(t)$ can fail to be positive for all time for some $x(0)>0$ and some such $k_{1}$ or $k_{5}$ with sufficiently large absolute values.

Remark 5.2. It is required for modeling coherency that both the epidemic SEIR model and its observer be positive dynamic systems. The condition of nonnegativeness of $(b+B(t) \widehat{x}(t))$ for all time in Assertion 5.1 requires $g \geq 0$, and

$$
(\widehat{\mu}-g) N \geq\left(k_{1}+k_{5} \widehat{I}(t)\right) \widehat{S}(t)+k_{2} \widehat{E}(t)+k_{3} \widehat{I}(t)+k_{4} \widehat{R}(t) \geq-g N ; \quad \forall t \in \mathbf{R}_{0+}
$$

which may be guaranteed by choosing the controller gains under the knowledge $N=$ $\sum_{i=1}^{4} \widehat{x}_{i}(t)$, for all $t \in \mathbf{R}_{0+}$ Also, $\widehat{A}_{0}$ has to be a Metzler matrix, $\min _{t \in \mathbf{R}_{0^{+}}} \Delta \widehat{A}(t)>0$ and $\widehat{b}>0$ (Assertion 5.1(i)) so that $0 \leq g \leq \widehat{\mu}, k_{2} \leq 0,-\widehat{\gamma} \leq k_{3} \leq 0,0 \leq k_{4} \leq \widehat{\omega}, k_{1}+k_{5} \widehat{I}_{r} \geq 0$ in order that the observer be a positive system

$$
\left(\widehat{\beta}_{1}+k_{5}\right)\left(\widehat{I}_{r}-\max _{t \in \mathbf{R}_{0+}} \widehat{I}(t)\right) \geq 0 ; \quad k_{5}\left(\min _{t \in \mathbf{R}_{0+}} \widehat{I}(t)-\widehat{I}_{r}\right) \geq 0
$$

This restricts the generality of the choice of the gains in the vaccination control law (3.3) since it would be needed to accomplish with the control gain constraint $0 \geq k_{5} \geq-\beta_{1}$. However, if the requirement for the observer to be positive is removed then it is only needed that the SEIR model (2.1)-(2.4) be positive under a modified vaccination law (3.3)

$$
V(t)=\frac{1}{\widehat{\mu} N}\left(k_{1} \widehat{S}(t)+k_{2} \widehat{E}(t)+k_{3} \widehat{I}(t)+k_{4} \widehat{R}(t)+k_{5} \widehat{S}(t) \widehat{I}(t)+g N\right)
$$

by requiring the weaker condition that $0 \leq g \leq \widehat{\mu}$ and

$$
\min (\sigma, \omega, \gamma) \geq 0 ; \quad I_{r} \geq \max _{t \in \mathbf{R}_{0+}} I(t)
$$

Note that while Assertion 5.1(i) is of sufficiency type to guarantee positivity, the lack of all the joint above conditions in Assertion 5.1(ii) refers to a necessary condition for positivity in such cases. Note also that positive and total population equal to $N$ for all time implies necessary global stability so that we have directly the following.

Assertion 5.3. If Assertion 5.1(i) holds then the extended SEIR model (i.e., the combined SEIR model plus its observer) is globally stable if all the initial populations and their estimates are nonnegative. Furthermore all the susceptible, infected, infectious, and immune populations and their estimates are upperbounded by $N$ and the sum of all the populations and that of their estimates is equal to $N$ at any time. The converse is not true, in general, so that if the extended SEIR model is stable under Assertion 4.3 then such a model is not necessarily positive. 
The positivity of the observer-based vaccination control is a nonnegative function if the conditions (5.5) hold, guaranteeing that the observer is a positive system and furthermore the gain $0 \geq k_{5} \geq \max \left(-\beta_{1},-I_{r} / k_{1}\right) \geq-I(t) / k_{1}$. Thus, one has the following result.

Assertion 5.4. The vaccination control below is nonnegative for all time if $k_{5} \geq 0$ and (5.5) hold:

$$
V(t)=\frac{1}{\widehat{\mu} N}\left(k_{1} \widehat{S}(t)+k_{3} \widehat{I}(t)+k_{4} \widehat{R}(t)+k_{5} \widehat{S}(t) \widehat{I}(t)+g N\right)
$$

with $-\widehat{\gamma} \leq k_{3} \leq 0$. Furthermore, the observer is a positive system under the vaccination control (5.8). If, in addition, (5.7) holds then the SEIR model (2.1)-(2.4) is also a positive system under the vaccination control (5.8).

The more general vaccination control (3.3), namely,

$$
V(t)=\frac{1}{\widehat{\mu} N}\left(k_{1} \widehat{S}(t)+k_{2} \widehat{E}(t)+k_{3} \widehat{I}(t)+k_{4} \widehat{R}(t)+k_{5} \widehat{S}(t) \widehat{I}(t)+g N\right)
$$

with $k_{i} \geq 0, i=2,3,4$, but not jointly zero, $k_{5} \geq 0$, and subject to (5.5)-(5.7) is not guaranteed to be nonnegative for all time (since the observer is not guaranteed to be a positive system).

The following vaccination nonnegative control combined of (3.3) and (5.8) may be used when the positivity of the observer is not imposed:

$$
\begin{aligned}
& V(t)= \begin{cases}\bar{V}(t), & \text { if } 1 \geq \bar{V}(t) \geq 0, \\
\frac{1}{\widehat{\mu} N}\left(k_{1} \widehat{S}(t)+k_{4} \widehat{R}(t)+k_{5} \widehat{S}(t) \widehat{I}(t)+g N\right), & \text { otherwise, }\end{cases} \\
& \bar{V}(t):=\frac{1}{\widehat{\mu} N}\left(k_{1} \widehat{S}(t)+k_{2} \widehat{E}(t)+k_{3} \widehat{I}(t)+k_{4} \widehat{R}(t)+k_{5} \widehat{S}(t) \widehat{I}(t)+g N\right)
\end{aligned}
$$

with $k_{i} \geq 0, i=1,2,3,4,5$, subject to (5.5)-(5.7). This nonnegative vaccination control keeps simultaneously as positive systems to both the SEIR model and its observer.

\section{Combined Positivity, Stability, and Tracking Objective}

The problem at hand requires as tracking objective simultaneously stability, positivity and tracking in the sense that the immune population converges to the total population which means that the total population including susceptible, infected, and infectious populations is zero. Positivity implies global stability (while the converse is not always true) for all given combination of nonnegative initial populations but the suitable tracking objective is that the vaccination strategy leads asymptotically to a total immune population while the other populations converge to zero while keeping positivity and then global stability. This issue is now discussed through the design of the controller parameter $g$ whose appropriate design is the basis for achievement of asymptotic tracking. Note that in order that the SEIR model and its observer be both positive, it is needed that $0 \leq g \leq \widehat{\mu}$ under Assertion 5.1(i)—see Remark 5.2. 
The suitable tracking observer-based objective is $\widehat{R}(t) \rightarrow N$ as $t \rightarrow \infty$ what implies $\widehat{S}(t), \widehat{E}(t)$, $\widehat{I}(t) \rightarrow 0$ as $t \rightarrow \infty$ with $\|\tilde{x}(t)\|$ being as small as possible as $t \rightarrow \infty$. Define

$$
\widehat{b}_{1}:=\frac{\widehat{b}}{N}=(1,0,0,0)^{T} \widehat{\mu}+(-1,0,0,1)^{T} g=e_{1} \widehat{\mu}+\left(e_{4}-e_{1}\right) g
$$

where $e_{i}$ is the $i$ th unity vector in $\mathbf{R}^{4}$ with its $i$ th component being one. The above tracking objective may be achieved in some prescribed finite $T$ or infinite time (i.e., asymptotically as $T \rightarrow \infty)$. The asymptotic tracking objective is stated according to the relations of taking limits in (5.2) as $t \rightarrow \infty$ by designing a time-varying parameter $g \in P C^{(0)}\left(\mathbf{R}_{0+} ; \mathbf{R}\right)$ as follows:

$$
\begin{gathered}
g(t)= \begin{cases}\bar{g}(t) \quad \text { if } \bar{g}_{M} \geq \bar{g}(t) \geq 0,0 \leq t \leq T, \text { or if } \widehat{\mu} \geq \bar{g}(t) \geq 0, t>T \\
\bar{g}(f(t)), \quad \text { otherwise }\end{cases} \\
\bar{g}(t):=\frac{1-\int_{0}^{t} e_{4}^{T} e^{\widehat{A}_{0}(t-\tau)}\left(e_{1} \widehat{\mu}+N^{-1} \Delta \widehat{A}(\tau) \widehat{x}(\tau)\right) d \tau}{\int_{0}^{t} e_{4}^{T} e^{\widehat{A}_{0}(t-\tau)}\left(e_{4}-e_{1}\right) d \tau} \\
f(t):=\max \left(t_{1}<t: \bar{g}\left(t_{1}\right) \geq 0, g\left(t_{1}+\widetilde{t}\right)<0 ; \forall \tilde{t} \in\left(0, t-t_{1}\right]\right) \quad \text { if } 0 \leq t \leq T
\end{gathered}
$$

with $\widehat{\mu} \leq \bar{g}_{M}<\infty$ being a prefixed real constant which can be potentially large. It turns out the following main result.

Theorem 6.1. If $\widehat{A}_{0}$ is a stability matrix then $g(t) \rightarrow g_{\infty} \in[0, \widehat{\mu}]$. If the constant $g$ is replaced with the piecewise continuous function defined by (6.2) in the SEIR observer, (5.5) holds and the vaccination control (5.10) is used under nonnegative initial conditions of the observer then the SEIR observer is a positive system with all the populations being nonnegative and of total sum equal to $N$ for all time while the vaccination control is also nonnegative for all time. Furthermore, $\widehat{R}(t) \rightarrow N, \widehat{S}(t), \widehat{E}(t)$, $\widehat{I}(t) \rightarrow 0$ as $t \rightarrow \infty$.

If, in addition, (5.7) holds then the SEIR model is also a positive system with populations nonnegative and equalizing $N$ for all time. The estimation error fulfills the uniform upperbound through time of Assertion 4.4.

\section{Simulation Examples}

This section illustrates through simulation examples the theoretical results stated in the previous sections for the combined SEIR control-observer model. The first example is concerned with the estimation properties of the observer given by (3.1) in the absence of vaccination. Then, the vaccination strategy given by (3.3) is introduced and its effects analyzed. The SEIR model is described by the following parameters taken from an influenza outbreak in an English college, also used in [19]:

$$
\frac{1}{\mu}=255 \text { days }, \quad \frac{1}{\sigma}=2.2 \text { days }, \quad \frac{1}{\omega}=15 \text { days }, \quad \beta=1.66 \text { days }^{-1}
$$




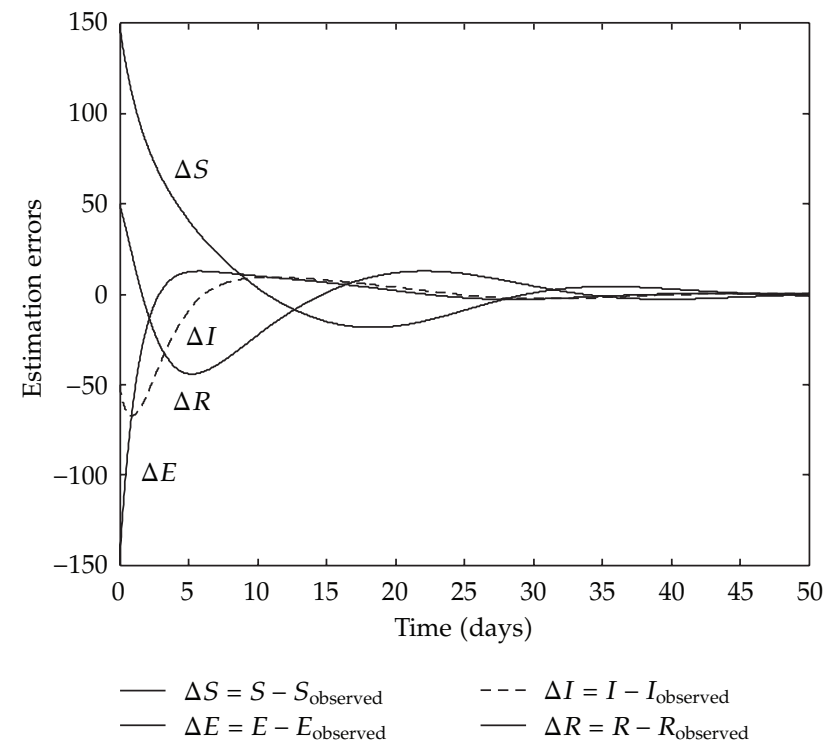

Figure 1: Observation error in the vaccination-free case and all the parameters known.

while it is taken $\gamma=\sigma$. The initial conditions are given by $S(0)=400, E(0)=150, I(0)=250$, and $R(0)=200$ individuals so that the total population is $N=N(0)=N(t)=1000$ individuals.

\subsection{Observer Vaccination-Free Dynamics}

Firstly, we consider the case when no vaccination is applied. The initial estimates of each population are $\widehat{S}(0)=250, \widehat{E}(0)=150, \widehat{I}(0)=150, \widehat{R}(0)=450$ individuals. Note that there is a great difference between the actual initial values and the estimated ones. Figure 1 shows the error between the actual and the estimated variables through time in the case when all the parameters of the model are known.

As it can be seen in Figure 1, all the observation errors asymptotically vanish and the observer is capable of identifying the steady state values of the populations. Figure 2 shows, as a matter of example, the time evolution of the susceptible and immune while Figure 3 shows the evolution of each population.

However, when the parameters of the system are not known and an estimation of its value is used, the observer does not track the real populations as shown in Figure 4 with estimated parameters

$$
\frac{1}{\widehat{\mu}}=235 \text { days }, \quad \frac{1}{\widehat{\sigma}}=2 \text { days }, \quad \frac{1}{\widehat{\omega}}=14 \text { days }, \quad \widehat{\beta}=1.46 \text { days }^{-1}
$$

and $\hat{\gamma}=\widehat{\sigma}$.

Nevertheless, the observer-based control law (3.3) still works in the presence of this estimation error. 


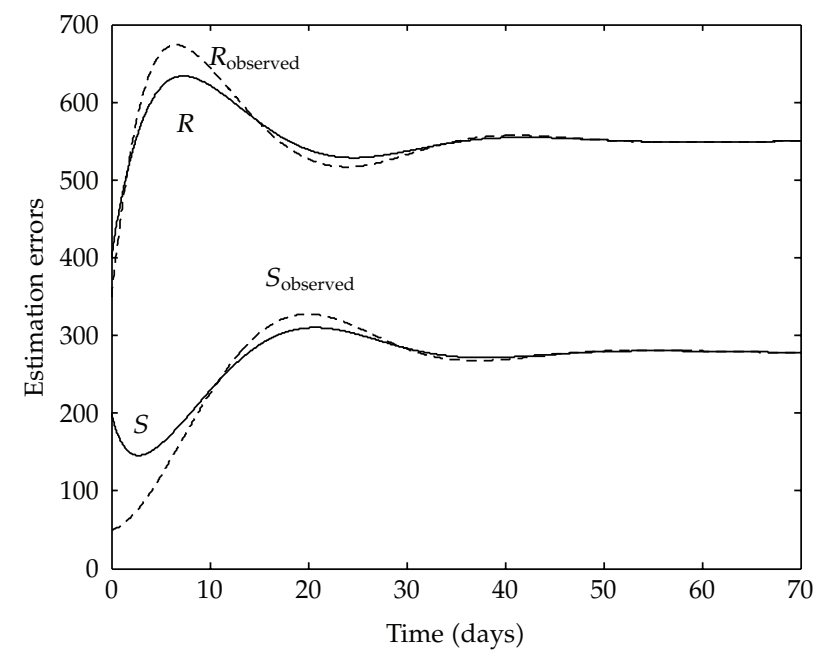

Figure 2: Evolution of the real and observed states for the susceptible and immune.

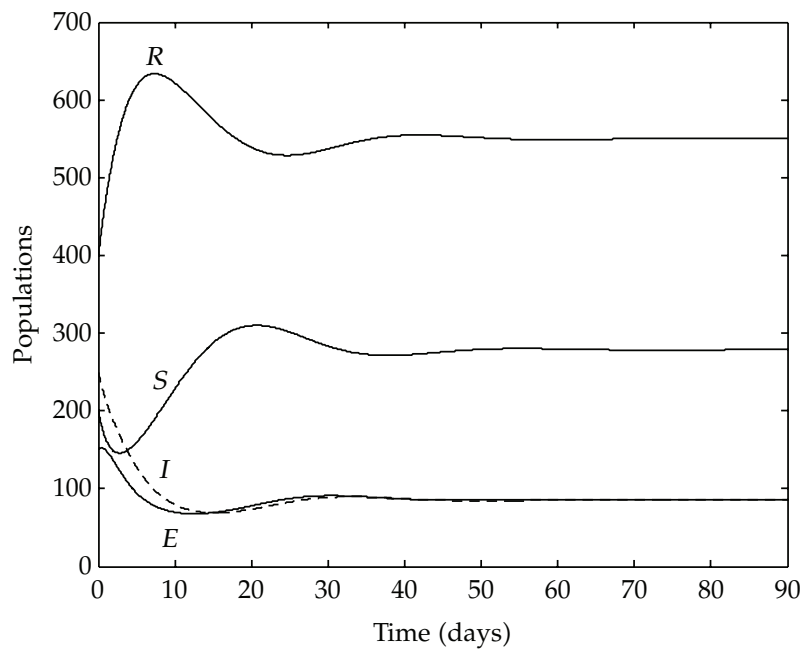

Figure 3: Evolution of the current populations through time.

\subsection{Combined Observer-SEIR Model Dynamics in the Presence of Vaccination}

In this section, a vaccination strategy given by (3.3) is introduced in the system. As commented in Section 5, Remark 5.2, it is desirable for modeling coherency that both, the SEIR model and its observer, be positive. Hence, this is the case considered in this simulation example. In order to guarantee the positiveness of both systems, the control gains have to be chosen according Assertions 4.4 and 5.1 from Section 5 with $k_{1}=1, k_{2}=-0.1<0, k_{3}=-\widehat{\gamma}, k_{4}=0.95 \widehat{\omega}$, $k_{5}=-\widehat{\beta}_{1}, g=\widehat{\mu}$ satisfying both assertions. Figures 5 and 6 show the time evolution of the populations and the observation error, respectively.

Figure 5 shows that not only the SEIR model is globally stable regardless the observation error depicted in Figure 6 but also that the observer-based control law erradicates 


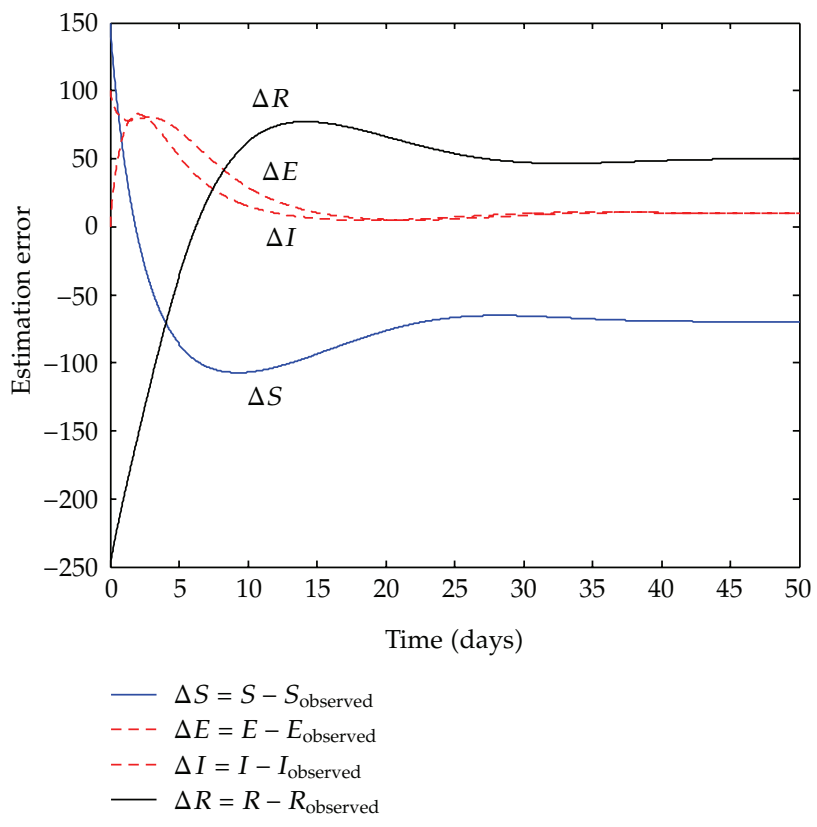

Figure 4: Observation error in the vaccination-free case and unknown model parameters.

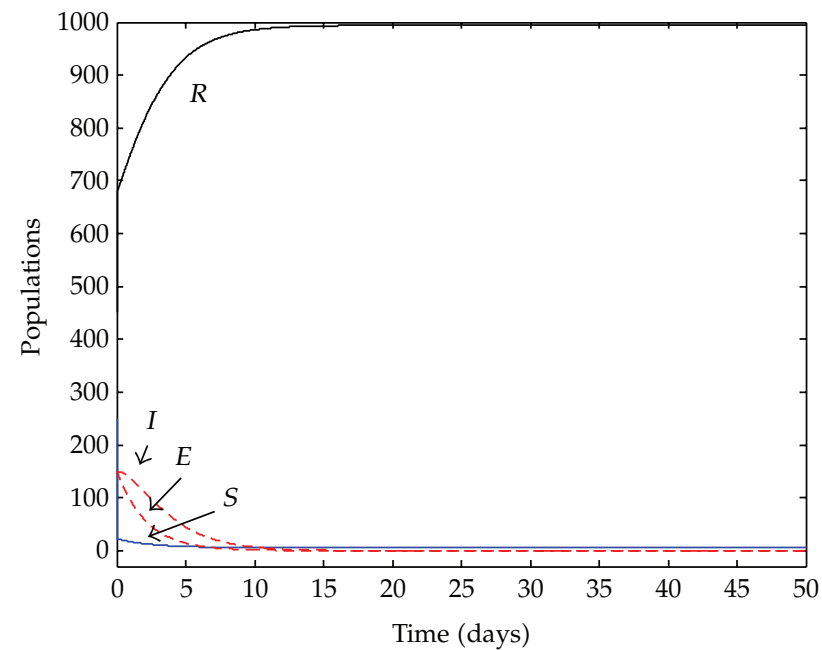

Figure 5: Evolution of the populations with vaccination and unknown parameters.

the infective and infectious while the immune almost reaches to be the total population $N$. A small number of the susceptible still appear in the steady state. This behavior is much better than the vaccination-free one depicted in Figure 3 where a number of the infective and infectious appear. Furthermore, the observed SEIR model is also positive as shown in Figure 7.

It is remarkable to notice the good results obtained by the proposed vaccination strategy making almost all the population become immune. Thus, it may not be necessary in practice to implement the complex time-varying gain given by (6.2) to make immune the total 


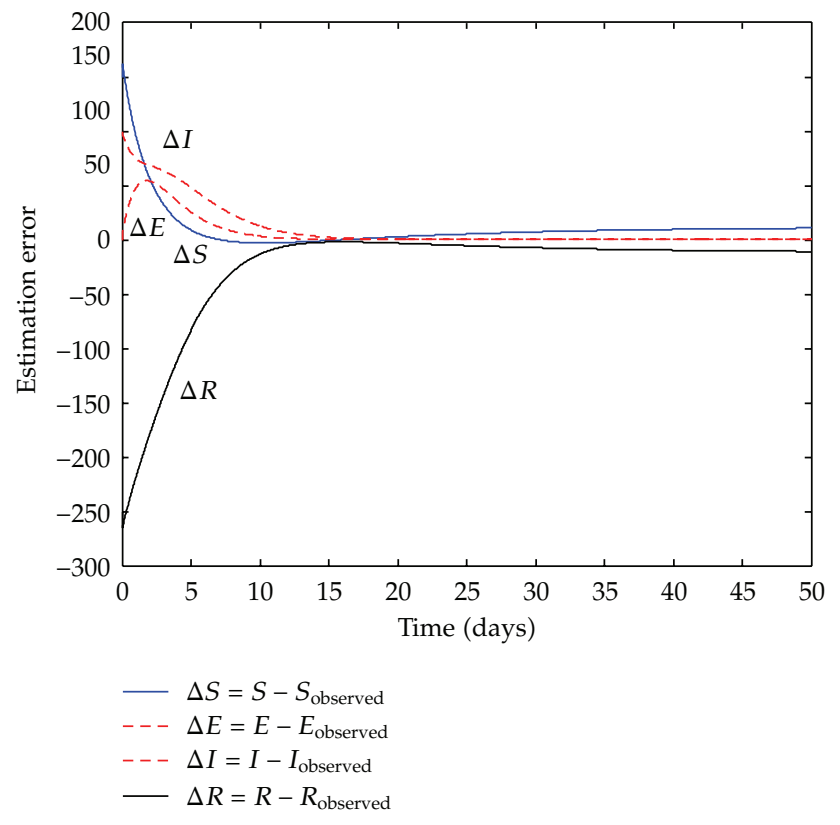

Figure 6: Observation error with vaccination and unknown parameters.

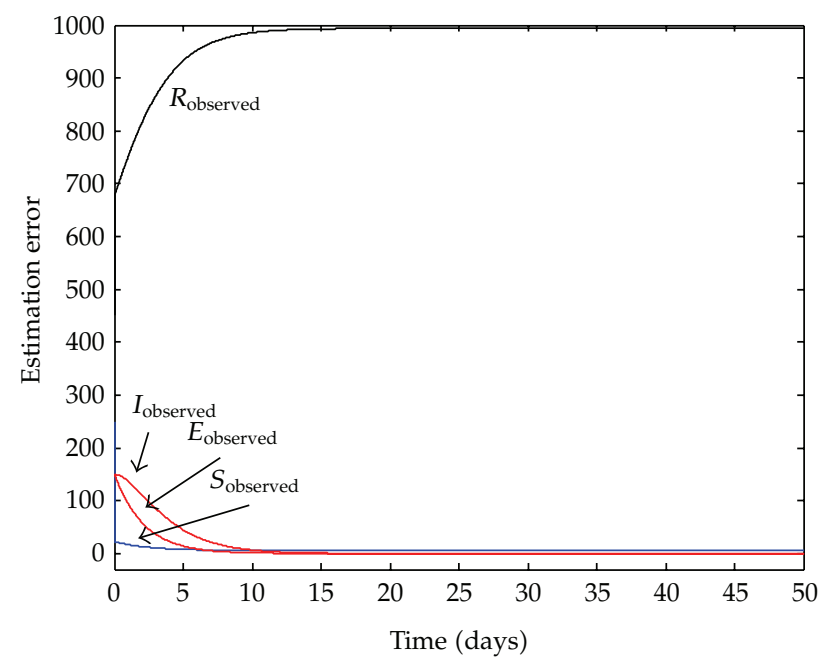

Figure 7: Time evolution of the positive observed states.

population. It suffices with taking $g=\widehat{\mu}$ which is the largest value within its admissible interval $g \in[0, \widehat{\mu}]$ according to Theorem 6.1 in Section 6 .

\section{Acknowledgments}

The authors thank the Spanish Ministry of Education for its support of this work through Grant DPI2009-07197 and the Basque Government for its support through Grants IT378-10 and SAIOTEK SPE07UN04. 


\section{References}

[1] M. De la Sen and S. Alonso-Quesada, "A control theory point of view on Beverton-Holt equation in population dynamics and some of its generalizations," Applied Mathematics and Computation, vol. 199, no. 2 , pp. 464-481, 2008.

[2] M. De la Sen and S. Alonso-Quesada, "Control issues for the Beverton-Holt equation in ecology by locally monitoring the environment carrying capacity: non-adaptive and adaptive cases," Applied Mathematics and Computation, vol. 215, no. 7, pp. 2616-2633, 2009.

[3] M. De La Sen and S. Alonso-Quesada, "Model-matching-based control of the Beverton-Holt equation in ecology," Discrete Dynamics in Nature and Society, vol. 2008, Article ID 793512, 21 pages, 2008.

[4] M. De La Sen, "About the properties of a modified generalized Beverton-Holt equation in ecology models," Discrete Dynamics in Nature and Society, Article ID 592950, 23 pages, 2008.

[5] M. De la Sen, "The generalized Beverton-Holt equation and the control of populations," Applied Mathematical Modelling, vol. 32, no. 11, pp. 2312-2328, 2008.

[6] D. Mollison, Epidemic Models: Their Structure and Relation to Data, Newton Institute, Cambridge University Press, 2003.

[7] M. J. Keeling and P. Rohani, Modeling Infectious Diseases in Humans and Animals, Princeton University Press, Princeton, NJ, USA, 2008.

[8] O. D. Makinde, "Adomian decomposition approach to a SIR epidemic model with constant vaccination strategy," Applied Mathematics and Computation, vol. 184, no. 2, pp. 842-848, 2007.

[9] P. Yongzhen, L. Shuping, L. Changguo, and S. Chen, "The effect of constant and pulse vaccination on a SIR epidemic model with infectious period," Applied Mathematics and Computation, vol. 35, no. 8, pp. 3866-3878, 2011.

[10] N. Ortega, L. C. Barros, and E. Massad, "Fuzzy gradual rules in epidemiology," Kybernetes, vol. 32, no. 3-4, pp. 460-477, 2003.

[11] H. Khan, R. N. Mohapatra, K. Vajravelu, and S. J. Liao, "The explicit series solution of SIR and SIS epidemic models," Applied Mathematics and Computation, vol. 215, no. 2, pp. 653-669, 2009.

[12] X. Song, Y. Jiang, and H. Wei, "Analysis of a saturation incidence SVEIRS epidemic model with pulse and two time delays," Applied Mathematics and Computation, vol. 214, no. 2, pp. 381-390, 2009.

[13] T. Zhang, J. Liu, and Z. Teng, "Dynamic behavior for a nonautonomous SIRS epidemic model with distributed delays," Applied Mathematics and Computation, vol. 214, no. 2, pp. 624-631, 2009.

[14] B. Mukhopadhyay and R. Bhattacharyya, "Existence of epidemic waves in a disease transmission model with two-habitat population," International Journal of Systems Science, vol. 38, no. 9, pp. 699$707,2007$.

[15] J. Zhang and Z. Jin, "The analysis of epidemic network model with infectious force in latent and infected period," Discrete Dynamics in Nature and Society, vol. 2010, Article ID 604329, 12 pages, 2010.

[16] W. Wang, J. Xin, and F. Zhang, "Persistence of an SEIR model with immigration dependent on the prevalence of infection," Discrete Dynamics in Nature and Society, vol. 2010, Article ID 727168, 7 pages, 2010.

[17] Y. Ma, B. Liu, and W. Feng, "Dynamics of a birth-pulse single-species model with restricted toxin input and pulse harvesting," Discrete Dynamics in Nature and Society, vol. 2010, Article ID 142534, 20 pages, 2010.

[18] R. J. Villanueva, A. J. Arenas, and G. González-Parra, “A nonstandard dynamically consistent numerical scheme applied to obesity dynamics," Journal of Applied Mathematics, vol. 2008, Article ID 640154 14 pages, 2008.

[19] M. De la Sen and S. Alonso-Quesada, "A simple vaccination control strategy for the SEIR epidemic model," in Proceedings of the 5th IEEE International Conference on Management of Innovation and Technology, pp. 1037-1044, June 2010.

[20] J. Tervo, M. T. Nihtilä, and P. Kokkonen, “On controllability, parametrization, and output tracking of a linearized bioreactor model," Journal of Applied Mathematics, no. 5, pp. 243-276, 2003.

[21] V. Azhmyakov, "Convexity of the set of fixed points generated by some control systems," Journal of Applied Mathematics, vol. 2009, Article ID 291849, 14 pages, 2009.

[22] L. Pacheco and N. S. Luo, "Mobile local trajectory tracking with dynamic model predictive control techniques," International Journal of Innovative Computing Information and Control, vol. 7, no. 6, pp. 3457-3483, 2011.

[23] P. Balaguer, "Similar model reference adaptive control with bounded control effort," International Journal of Adaptive Control and Signal Processing, vol. 25, no. 7, pp. 577-592, 2011.

[24] M. De la Sen, "Online optimization of the free parameters in discrete adaptive-control systems," IEE Proceedings D, vol. 131, no. 4, pp. 146-157, 1984. 
[25] M. De la Sen, R. P. Agarwal, A. Ibeas, and S. Alonso-Quesada, “On a generalized time-varying SEIR epidemic model with mixed point and distributed time-varying delays and combined regular and impulsive vaccination controls," Advances in Difference Equations, vol. 2010, Article ID 281612, 42 pages, 2010.

[26] M. De la Sen, R. P. Agarwal, A. Ibeas, and S. Alonso-Quesada, “On the existence of equilibrium points, boundedness, oscillating behavior and positivity of a SVEIRS epidemic model under constant and impulsive vaccination," Advances in Difference Equations, vol. 2011, Article ID 748608, 32 pages, 2011.

[27] N. Zhang, F. Chen, Q. Su, and T. Wu, "Dynamic behaviors of a harvesting Leslie-Gower predator-prey model," Discrete Dynamics in Nature and Society, vol. 2011, Article ID 473949, 14 pages, 2011.

[28] H. Lu and W. Wang, "Dynamics of a nonautonomous Leslie-Gower type food chain model with delays," Discrete Dynamics in Nature and Society, vol. 2011, Article ID 380279, 19 pages, 2011.

[29] M. De la Sen, "On impulsive time-varying systems with unbounded time-varying point delays: stability and compactness of the relevant operators mapping the input space into the state and output spaces," The Rocky Mountain Journal of Mathematics, vol. 37, no. 1, pp. 79-129, 2007.

[30] S. Alonso-Quesada and M. De la Sen, "Robust adaptive control of discrete nominally stabilizable plants," Applied Mathematics and Computation, vol. 150, no. 2, pp. 555-583, 2004.

[31] M. De la Sen, "On positivity and stability of a class of time-delay systems," Nonlinear Analysis, Real World Applications, vol. 8, no. 3, pp. 749-768, 2007.

[32] M. De la Sen, "On positivity of singular regular linear time-delay time-invariant systems subject to multiple internal and external incommensurate point delays," Applied Mathematics and Computation, vol. 190, no. 1, pp. 382-401, 2007. 


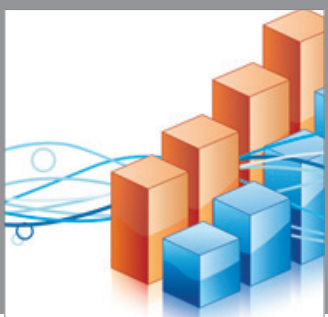

Advances in

Operations Research

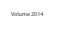

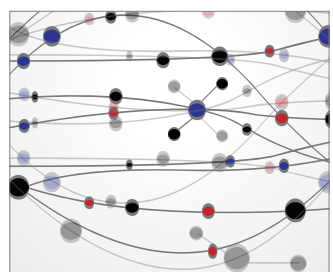

\section{The Scientific} World Journal
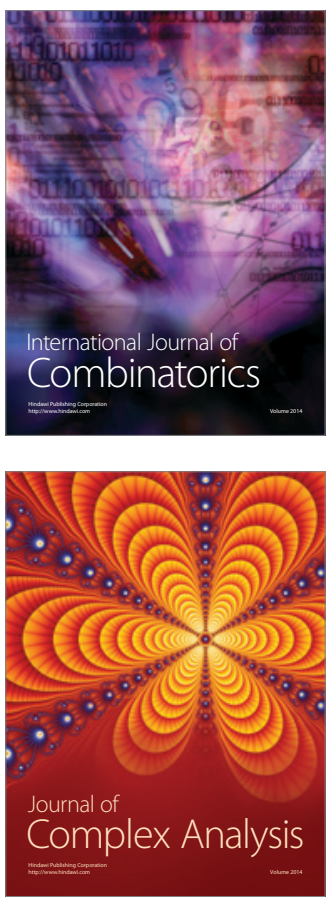

International Journal of

Mathematics and

Mathematical

Sciences
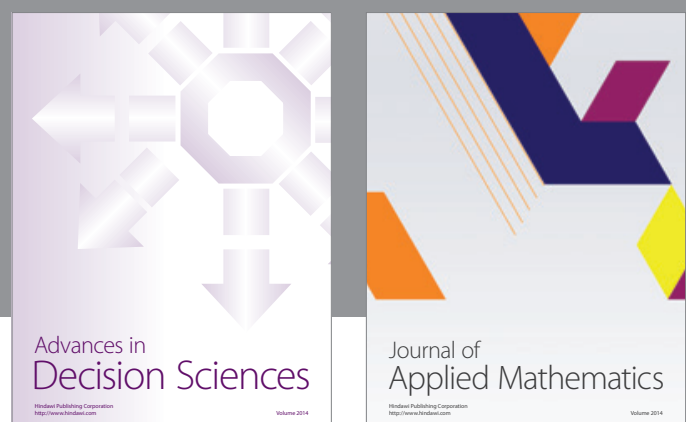

Journal of

Applied Mathematics
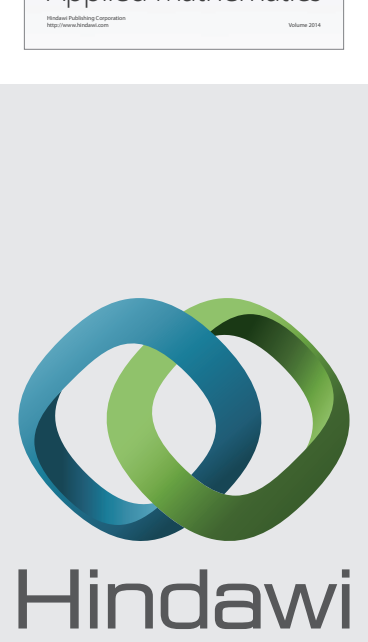

Submit your manuscripts at http://www.hindawi.com
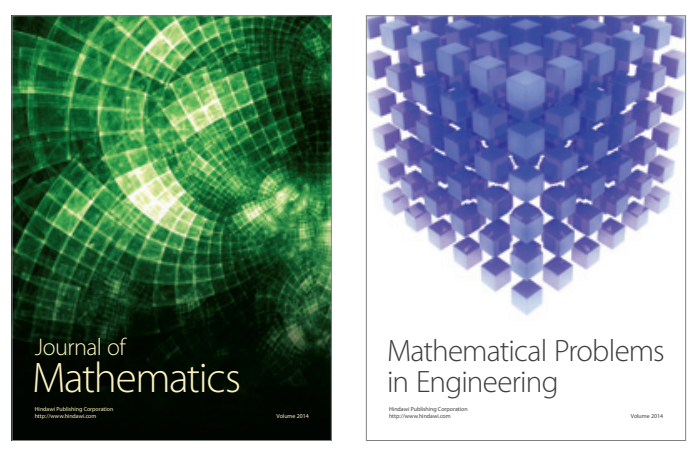

Mathematical Problems in Engineering
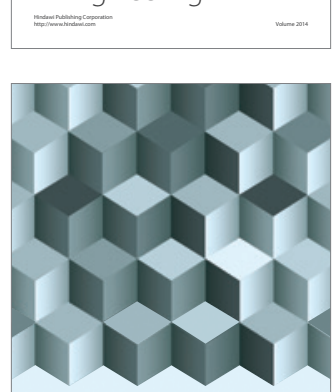

Journal of

Function Spaces
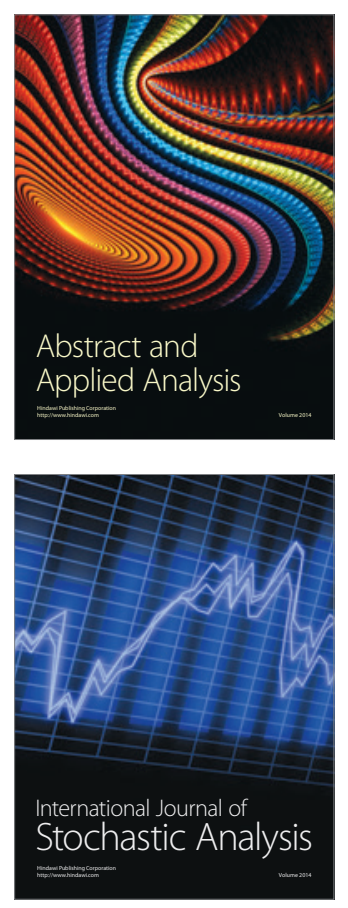

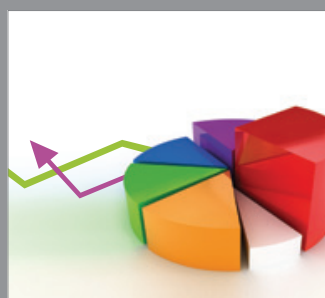

ournal of

Probability and Statistics

Promensencen
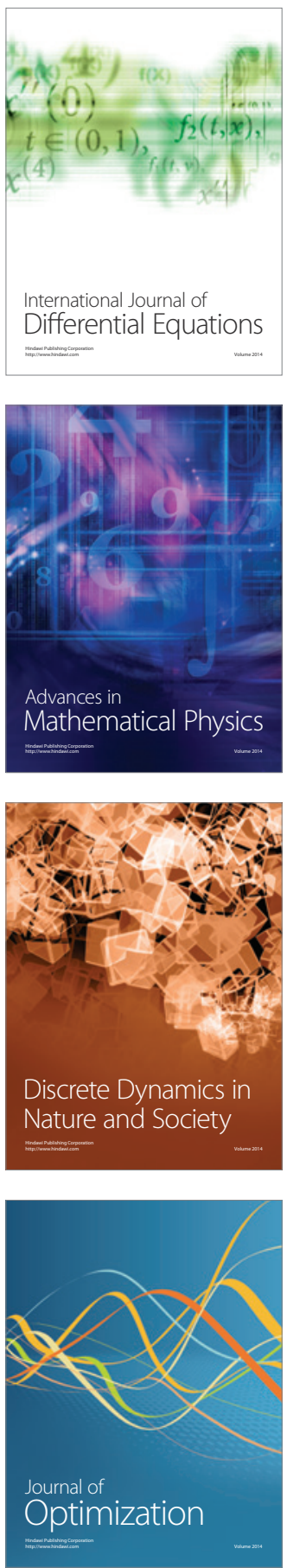